RICYDE. Revista Internacional de Ciencias del Deporte doi: $10.5232 /$ ricyde

Rev. int. cienc. deporte

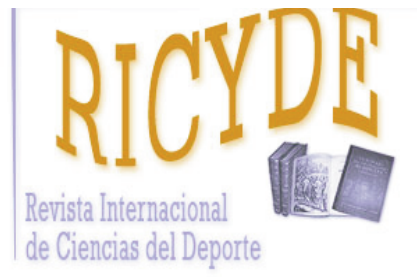

RICYDE. Revista Internacional de Ciencias del Deporte VOLUMEN XIII - AÑO XIII

Páginas:117-138 ISSN:1885-3137

Número 48 - Abril - 2017

\title{
Revisión de los factores de riesgo y los programas de prevención de la lesión del ligamento cruzado anterior en fútbol femenino: propuesta de prevención Review risk factors and prevention programs of the anterior cruciate ligament injury in female football: prevention proposal
}

\author{
Blanca Romero-Moraleda, Ángel Cuéllar, Jaime González, Nerea Bastida, Elsa Echarri, \\ Jana Gallardo y Víctor Paredes \\ Facultad de Salud. Universidad Camilo José Cela. España
}

\section{Resumen}

Los objetivos de esta revisión fueron analizar las causas y factores de riesgo de la lesión del LCA, realizar una revisión sistemática de los programas de prevención actuales y proponer un protocolo para la prevención de la lesión del LCA. Para ello, se realizó una búsqueda en las bases de datos PuBmed, PeDro y EBSCO, teniendo en cuenta palabras clave en términos DeCS y MeSH, los cuales aportaron 606 y 228 artículos respectivamente. De acuerdo a los criterios de inclusión y exclusión, se seleccionan 18 artículos para ser estudiados. En cuanto a los factores de riesgo de cualquier lesión de rodilla son: lesión previa, fatiga muscular y desequilibrio de fuerza entre isquiosurales y cuádriceps (McCall y col., 2014). Específicamente para la lesión del LCA las causas pueden ser el ángulo $\mathrm{Q}$, el valgo de rodilla, el genu recurvatum, el pie pronador y la torsión externa de la tibia. Además el LCA de la mujer es menor en longitud y en sección transversal que el del hombre (Sutton y col., 2013). Los programas de prevención que han mostrado disminuir la frecuencia lesional inciden en el trabajo del core, la propiocepción, el control motor, el aprendizaje de la técnica de amortiguación, la flexibilidad y el trabajo excéntrico (McCall y col., 2014). En conclusión, se exponen los factores de riesgo específicos a esta lesión en mujeres junto a la eficacia mostrada en los diferentes programas preventivos analizados. Los programas de prevención incluyen el trabajo de la fuerza neuromuscular, estiramientos, pliometría, equilibrio, core y corrección de la técnica de amortiguación del salto y los cambios de dirección.

Palabras clave: ligamento cruzado anterior; fútbol femenino; factores de riesgo; programas de prevención.

\section{Abstract}

The aims of this review were to analyce the causes and risk factors for ACL injury, a systematic review of current prevention programs and pose a protocol for preventing of ACL injury. Searches were performed in PubMed, PeDRO, and EBSCO data, taking into account keywords in DeCS and MeSH terms, which provided with 606 and 228 articles respectively. According to inclusion and exclusion criteria, 18 articles were selected for study. For any knee injury risk factors are a previous injury, fatigue and muscle strength imbalance between hamstrings and the quadriceps muscle (McCall et al., 2014). In the case of ACL injury studies focus on causes like the $Q$ angle, the valgus knee, the genu recurvatum, the pronator foot and the external tibial torsion among others. In addition, the ACL of women is smaller in length and has smaller cross section than the ACL of men (Sutton $y$ Bullock, 2013). Prevention programs included training the core, propioception, flexibility, motor control, learn technique and eccentric work (McCall et al., 2014; Myer et al., 2012; Stevenson et al., 2014; Sugimoto et al., 2014). In conclusion, the specific risk factors for this injury in women are presented with the effectiveness shown in the different preventive programs analyzed. The prevention programs analyzed include improvements for neuromuscular strength, stretching, plyometry, balance, core and correction of the technique of jump landing and changes of direction.

Key words: anterior cruciate ligament $(A C L)$; female football; risk factors; prevention programs. 
Romero-Moraleda, B.; Cuéllar, A.; González, J.; Bastida, N.; Echarri, E.; Gallardo, J., y Paredes, V. (2017). Revisión de los factores de riesgo y los programas de prevención de la lesión del ligamento cruzado anterior en fútbol femenino: propuesta de prevención. RICYDE. Revista internacional de ciencias del deporte, 48(13), $117-138$. https://doi.org/10.5232/ricyde2017.04803

\section{Introducción}

$\mathrm{L}$ a lesión del ligamento cruzado anterior (LCA) de la rodilla incapacita de forma inmediata necesitando un largo período de recuperación hasta la vuelta a la competición independientemente del tratamiento elegido (conservador frente a quirúrgico).

La lesión del LCA es la más común dentro de las lesiones de rodilla (Pauzenberger, Syré y Schurz, 2013), suponiendo más de un 50\% de las lesiones de esta articulación (Joseph y col., 2012), pudiendo llegar a alcanzar hasta el 90\% (Woo, Abramowitch, Kilger y Liang, 2006). También esta lesión tiene mayor incidencia en fútbol frente a otros deportes de equipo como el baloncesto o el voleibol y con mayor frecuencia en mujeres que en hombres (Joseph y col., 2012). Frecuentemente están asociadas a otras lesiones articulares concomitantes y provocan un aumento del riesgo de artrosis precoz independientemente del tratamiento administrado (Lohmander, Östenberg, Englund y Roos, 2004).

Un estudio epidemiológico que analiza los principales mecanismos de lesión de LCA muestra que el 53\% se produjeron realizando un cambio de dirección al lado de la rodilla lesionada ( $10 \%$ realizando también cambio dirección pero al lado contrario) y el $26 \%$ aterrizando, tras realizar un golpeo de cabeza (Faunø y Wulff, 2006). El dato más relevante a destacar de este estudio es que en todos los casos, en el momento de la lesión, el pie se encontraba en contacto con el suelo y en el $90 \%$ de las lesiones de LCA, se produjeron sin contacto con otro jugador ( $57 \%$ en contacto con balón pero no con otro jugador y $39 \%$ sin contacto con balón ni con otro jugador). Otro estudio epidemiológico nos muestra que el ratio de lesión LCA es mayor en la práctica de fútbol frente a otros deportes como baloncesto, voleibol, etc. y mayor incidencia en mujeres que en hombres (Joseph y col., 2012). Varios estudios comparan la incidencia de la lesión de LCA en hombres y mujeres demostrando que el género femenino tiene unos ratios de incidencia entre tres y ocho veces mayor que el masculino y unos ratios de 20,8 mayores en competición que durante el entrenamiento (Woo y col,. 2006; Joseph y col,. 2012; Beynnon y col., 2014).

A pesar de la creciente investigación acerca de los factores de riesgo asociados a sufrir la lesión del LCA, la prevalencia de esta lesión en los últimos años está aumentado en mujeres (Grimm y Kirkendall, 2007).

Debido a la alta incidencia de esta lesión así como al tiempo de retirada deportiva que supone, es necesario abordar los factores de riesgo específicos de esta lesión haciendo hincapié en las diferencias que hay por género. Esto junto a la revisión de los programas de prevención que han mostrado ser eficaces para disminuir el riesgo de lesión, nos permitirá realizar propuestas de prevención específicas en mujeres futbolistas extrapolables a otros deportes de equipo.

Por ello, el objetivo de este estudio es analizar las causas y factores de riesgo de la lesión del LCA y realizar una revisión sistemática de los programas de prevención actuales para la lesión del LCA.

\section{Material y métodos}

Se realizó una búsqueda exhaustiva en las bases de datos Pubmed, PeDro y Google Scholar, teniendo en cuenta palabras clave en términos DeCS y MeSH (descriptores en Ciencias de la Salud).

La búsqueda se limitó a artículos de lengua inglesa o castellana a partir del año 1999 y estudios en humanos. Principalmente las palabras usadas fueron "prevención de lesiones", "ligamento cruzado anterior" "LCA" "mujeres" ("injury prevention" "anterior cruciate ligament" "ACL" "female"); con el conector AND. 
Romero-Moraleda, B.; Cuéllar, A.; González, J.; Bastida, N.; Echarri, E.; Gallardo, J., y Paredes, V. (2017). Revisión de los factores de riesgo y los programas de prevención de la lesión del ligamento cruzado anterior en fútbol femenino: propuesta de prevención. RICYDE. Revista internacional de ciencias del deporte, 48(13), $117-138$. https://doi.org/10.5232/ricyde2017.04803

El resultado de cada una de las búsquedas se muestra en la tabla 1. Para la selección de estudios se establecieron los siguientes criterios de inclusión: artículos publicados entre 1999 y 2016, cuyo diseño de estudio sean ensayos clínicos, revisiones sistemáticas y meta-análisis en inglés o castellano. La figura 1 muestra el flujograma de los estudios incluidos tras la evaluación de la calidad metodológica, a través de la lista de chequeo Consort para ensayos clínicos y Estrategia PICO para las revisiones sistemáticas. Los artículos del motor de búsqueda PEdro fueron evaluados también evaluados por los 11 items incluidos en la escala PEdro.

Tabla 1. Resultados de la búsqueda

\begin{tabular}{|c|c|c|}
\hline Base de datos & Términos de búsqueda & Número de resultados \\
\hline Google Académico & $\begin{array}{l}\text { Prevención de lesiones + ligamento } \\
\text { cruzado anterior }+ \text { mujeres }\end{array}$ & 1.450 \\
\hline Google Scholar & \multirow{4}{*}{$\begin{array}{l}\text { "injury prevention" and "anterior } \\
\text { cruciate ligament" or " } A C L \text { " and } \\
\text { "female" OR "women" OR "girl" }\end{array}$} & 20.800 \\
\hline Pubmed & & 7.994 \\
\hline PEdro & & 21 \\
\hline Teseo & & 41 \\
\hline
\end{tabular}

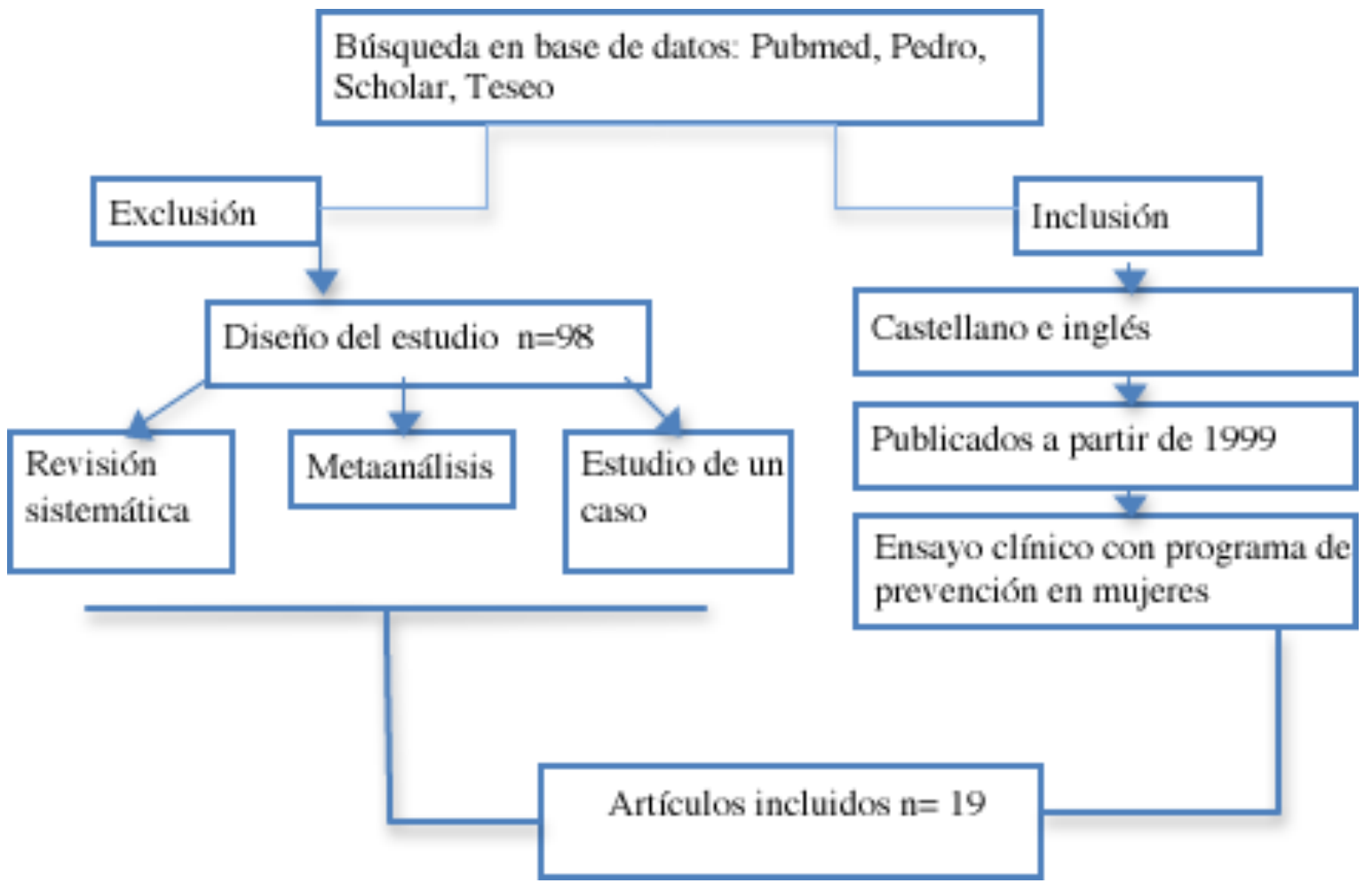

Figura 1. Flujograma de la búsqueda 
Romero-Moraleda, B.; Cuéllar, A.; González, J.; Bastida, N.; Echarri, E.; Gallardo, J., y Paredes, V. (2017). Revisión de los factores de riesgo y los programas de prevención de la lesión del ligamento cruzado anterior en fútbol femenino: propuesta de prevención. RICYDE. Revista internacional de ciencias del deporte, 48(13), $117-138$. https://doi.org/10.5232/ricyde2017.04803

\section{Resultados y discusión}

En este apartado se muestra por un lado, los estudios que han analizado los factores de riesgo de la lesión del LCA, tabla 2 y por otro lado, la evidencia científica de los programas de prevención para disminuir el riesgo de lesión del LCA, tabla 3, mostrandose los diferentes estudios que han tratado de comprobar la efectividad que tiene un programa de prevención específico para disminuir el riesgo de lesión.

En este estudio de revisión se recogen cuales son los factores de riesgo más asociados a la lesión del LCA que presentan las mujeres deportistas, especialmente en fútbol femenino. Además se han analizado los resultados que presentan los diferentes programas de prevención cuyo objetivo es disminuir el riesgo de lesión.

Previo a la descripción de los factores de riesgo mostrados en la tabla 2 del apartado resultados es necesario describir la biomecánica del LCA para comprender los mecanismos de lesión más comunes.

Biomecanicamente, la principal función del LCA es evitar la anteriorización de la tibia con respecto al fémur y secundariamente controlar los movimientos en varo, valgo y rotación de la rodilla. Presenta un comportamiento viscoelástico para disminuir la tensión de las deformaciones bruscas y relajarse en las deformaciones prolongadas (Kwan, Lin y Woo, 1993). En cuanto a su función propioceptora son las terminaciones de Ruffini y los corpúsculos de Pacini los encargados de recibir la información mecánica (Schutte, Dabezies, Zimny y Happel, 1987).

En referencia a la descripción del mecanismo de lesión conlleva momentos de traslación anterior de la tibia con rotación y valgo de rodilla que ocurre mayoritariamente en un pivotaje lateral (cambio de dirección) junto con un aterrizaje o desaceleración de la acción deportiva (Quatman y Hewett, 2009). Además de conocer el mecanismo de lesión es importante conocer los factores de riesgo para individualizar especificamente los programas de prevención.

\section{Factores de riesgo}

Como se resumen en la tabla 2, ante cualquier lesión de rodilla, los tres factores de riesgo principales son: haber tenido una lesión previa (recidiva), la fatiga muscular y el desequilibrio entre la musculatura anterior y posterior del muslo (cuádriceps vs isquiosurales) (McCall y col., 2014; Stevenson y col., 2014; Thomas, Villwock, Wojtys y Palmieri-Smith, 2013). Numerosos estudios biomecánicos resaltan la importancia de este último factor de riesgo debido a que una contracción de la musculatura isquiosural junto con los abductores de cadera se encargan de la estabilización de la cadera en el momento de flexión y permite neutralizar la tendencia del torque del cuádriceps a la anteriorización de la tibia sobre el fémur (Elias y Mizner, 2014; MacLeod, Snyder-Mackler y Buchanan, 2014; Telianidis y col., 2014).

Tabla 2. Resumen de los factores de riesgo en la lesión de LCA

\begin{tabular}{|l|r|}
\hline Autor, año & Resultados: factores de riesgo \\
\hline McCall y col., 2014 & $\begin{array}{l}\text { - Lesión previa } \\
\text { - }\end{array}$ \\
& Fatiga muscular \\
\hline Bonci, 1999 & Desequilibrio de fuerza entre isquiosurales y cuádriceps \\
& - Desequilibrio de fuerzo entre isquiosurales y cuádriceps \\
& - Pie pronador \\
\hline
\end{tabular}


Romero-Moraleda, B.; Cuéllar, A.; González, J.; Bastida, N.; Echarri, E.; Gallardo, J., y Paredes, V. (2017). Revisión de los factores de riesgo y los programas de prevención de la lesión del ligamento cruzado anterior en fútbol femenino: propuesta de prevención. RICYDE. Revista internacional de ciencias del deporte, 48(13), $117-138$. https://doi.org/10.5232/ricyde2017.04803

\begin{tabular}{|c|c|c|}
\hline & & $\begin{array}{l}\text { Valgo de rodilla } \\
\text { Rotación externa de la tibia }\end{array}$ \\
\hline Griffin y col., 2005 & & $\begin{array}{l}\text { Pie pronador } \\
\text { Hiperextensión de rodilla } \\
\text { Valgo de rodilla } \\
\text { Rotación externa de la tibia } \\
\text { Ángulo Q } \\
\text { Debilidad glúteo medio }\end{array}$ \\
\hline Shult y col., 2010 & & $\begin{array}{l}\text { LCA más rígido en mujeres } \\
\text { Porcentaje de fibras de colágeno menor en mujeres } \\
\text { Debido al genu recurvatum de la mujer, aumenta la laxitud del } \\
\text { LCA } \\
\text { Días previos a la menstruación aumenta la laxitud del LCA } \\
\text { LCA de la mujer más pequeño en longitud y de menor sección } \\
\text { transversal }\end{array}$ \\
\hline Smith H.C. y col., 2012 (a) & & $\begin{array}{l}\text { Laxitud de rodilla } \\
\text { Hiperextensión de rodilla } \\
\text { Aumento del índice de masa corporal }\end{array}$ \\
\hline Smith H.C. y col., 2012 (b) & & $\begin{array}{l}\text { Lesión previa } \\
\text { Disminución del tiempo de reacción } \\
\text { Disminución de la velocidad de procesamiento } \\
\text { Disminución de memoria visual y verbal }\end{array}$ \\
\hline Hewett y col., 2005 & & $\begin{array}{l}\text { En lesión previa, a posteriori, aumenta el valgo de rodilla, la fuerza } \\
\text { de reacción con el suelo y disminuye el tiempo de amortiguación. } \\
\text { Desequilibrio neuromuscular: } \\
\text { ○ Dominancia ligamentosa: la musculatura no tiene la } \\
\text { capacidad de absorber las fuerzas y estas son transmitidas } \\
\text { a los ligamentos. } \\
\text { Dominancia del cuádriceps: esto se relaciona con menor } \\
\text { flexión de rodilla en la amortiguación y menor activación } \\
\text { de la musculatura posterior en la estabilización de la } \\
\text { rodilla. } \\
\text { Asimetría de activación en miembros inferiores: menor } \\
\text { activación muscular y control motor en la pierna no } \\
\text { dominante. } \\
\text { Dominancia del tronco: menor estabilidad lumbopélvica } \\
\text { conlleva menor capacidad de estabilización de la rodilla. }\end{array}$ \\
\hline Zazulat y col., 2007 & & $\begin{array}{l}\text { Déficit de control neuromuscular a nivel lumbopélvico está } \\
\text { asociado con inestabilidad cadera-rodilla y aumenta probabilidad } \\
\text { de lesión del LCA. }\end{array}$ \\
\hline Alentorn-Geli y col., 2009 (a) & & $\begin{array}{l}\text { Superficie de juego seca que aumenta la temperatura del césped y } \\
\text { aumenta la fricción. } \\
\text { Calzado de taco largo aumenta la fricción y por tanto el riesgo de } \\
\text { lesión. }\end{array}$ \\
\hline Alentorn-Geli y col., 2009 (b) & & $\begin{array}{l}\text { Menor tamaño de la escotadura intercondílea (más frecuente en } \\
\text { mujeres). }\end{array}$ \\
\hline
\end{tabular}


Romero-Moraleda, B.; Cuéllar, A.; González, J.; Bastida, N.; Echarri, E.; Gallardo, J., y Paredes, V. (2017). Revisión de los factores de riesgo y los programas de prevención de la lesión del ligamento cruzado anterior en fútbol femenino: propuesta de prevención. RICYDE. Revista internacional de ciencias del deporte, 48(13), $117-138$. https://doi.org/10.5232/ricyde2017.04803

\begin{tabular}{|c|c|}
\hline Hägglund y Waldén, 2015 & $\begin{array}{l}\text { - Porcentaje de lesiones del LCA: se cuantificaron } 21 \text { de } 4556 \\
\text { jugadoras entre } 12 \text { y } 17 \text { años: } \\
\circ \quad 76 \% \text { en partido } \\
\circ \quad 62 \% \text { sin contacto } \\
\circ \quad 52 \% \text { en la pierna no dominante } \\
\circ \quad 91 \% \text { en césped natural } \\
\circ \quad 48 \% \text { con } 15 \text { años de edad } \\
\circ \quad 62 \% \text { nacidas la primera mitad del año } \\
\circ \quad 95 \% \text { mujeres menárquicas } \\
\circ \quad 14 \% \text { lesión previa de rodilla } \\
\circ \quad 38 \% \text { con lesiones familiares previas del LCA }\end{array}$ \\
\hline Söderman y col., 2001 & $\begin{array}{l}\text { - } \quad \text { Registraron } 398 \text { lesiones del LCA: } \\
\quad \text { o } 86 \% \text { sucedieron en partido } \\
\circ \quad 52 \% \text { sin contacto } \\
\text { - } \quad 39 \% \text { participando en competición de edad senior siendo la } \\
\text { jugadora menor de } 16 \text { años. }\end{array}$ \\
\hline Yanguas-Leyes y col., 2011 & $\begin{array}{c}\text { - Registraron } 11 \text { lesiones del LCA de } 166 \text { casos: } \\
\circ \text { Mecanismo indirecto de lesión } \\
\circ \text { Mayor incidencia en partido }\end{array}$ \\
\hline
\end{tabular}

De los factores de riesgo asociados que destacamos de la tabla 2 son la pronunciación del valgo de rodilla, la hiperextensión de rodilla, el pie pronador, rotación externa de tibia y debilidad del glúteo medio, lo cual aumenta la báscula pélvica en el plano frontal y por tanto, el valgo de rodilla (Griffin y col., 2006; Mandelbaum y col., 2005). Otros factores de riesgo asociados son también el mayor ángulo Q y el aumento del Índice de Masa Corporal (IMC) (Acevedo, Rivera-Vega, Miranda y Micheo, 2014). El análisis y revisión de los factores de riesgo del LCA de Smith y col. nos señalan la importancia de los factores neuromusculares tales como: aumento del tiempo de reacción ante un estímulo, del tiempo de procesamiento y menor memoria verbal y visual (Smith y col., 2012). El trabajo de Hewet y col. (2010) nos destaca los posibles desequibrios musculares que aumentan el riesgo de sufrir la lesión de LCA (Hewett, Ford, Hoogenboom y Myer, 2010):

- Dominancia ligamentosa: la musculatura no tiene la capacidad de absorber las fuerzas y estas son transmitidas a los ligamentos puediendo aumentar el valgo de rodilla y la traslación anterior de la tibia.

- Dominancia del cuádriceps: esto se relaciona con menor flexión de rodilla en la amortiguación y menor activación de la musculatura posterior (isquiosurales, poplíteo, gastrocnemios) en la estabilización de la rodilla para evitar el desplazamiento anterior de la tibia.

- Asimetría de activación en miembros inferiores: menor activación muscular y control motor en la pierna no dominante, lo que conlleva una traslación del peso a la pierna dominante e inclinación lateral del tronco.

- Dominancia del tronco (activación insuficiente o con retardo de la musculatura lumbopélvica): menor estabilidad lumbopélvica conlleva menor capacidad de estabilización de la rodilla. 
Romero-Moraleda, B.; Cuéllar, A.; González, J.; Bastida, N.; Echarri, E.; Gallardo, J., y Paredes, V. (2017). Revisión de los factores de riesgo y los programas de prevención de la lesión del ligamento cruzado anterior en fútbol femenino: propuesta de prevención. RICYDE. Revista internacional de ciencias del deporte, 48(13), $117-138$. https://doi.org/10.5232/ricyde2017.04803

A nivel biomecánico y realizando una analogía del modelo de Panjabi, la estabilidad articular depende de la activación correcta del sistema activo muscular, del sistema pasivo articular (cápsula, ligamentos, meniscos y superficies articulares) y del adecuado control del sistema nervioso central que traduce la información recibida por los receptores articulares y musculares para devolver una respuesta adecuada que permita la establización articular (Panjabi, 1992). Por tanto, una adecuada propiocepción no es más que la adecuada integración de estos tres elementos para permitir integrar la información aferente tanto interna como externa y dar respuesta con la activación muscular adecuada para la estabilización articular.

En cuanto a las diferencias sexuales, los análisis de Sigward y col. (2012) donde compara las diferencias entre hombres y mujeres antes y después de la maduración sexual, muestra que las mujeres presentan un patrón de activación diferente en la recepción del salto. Tras la maduración sexual las mujeres aterrizan amortiguando menos, es decir, con menor flexión de cadera y rodilla y mayor momento de adución y rotación interna, provocando un aumento del valgo de rodilla, lo que somete a mayor estrés a la articulación de la rodila y al LCA (Sigward, Pollard y Powers, 2012). Además, en comparación con los hombres, en las mujeres hay una contracción predominante del cuádriceps respecto a la musculatura isquiosural (agonista o protectora del LCA) en el aterrizaje del salto y en las frenadas (movimientos excéntricos), lo que aumenta el riesgo de lesión del LCA al producir un desplazamiento anterior de la tibia (Shultz, Sander, Kirk y Perrin, 2005; Shultz, Nguyen y Schmitz, 2008; Sigward y col., 2012), a esto se le sumaría la diferencia estructural del LCA por género, encontrando que el LCA de las mujeres: (I) es más pequeño en longitud y con menor sección transversal que en hombres, (II) contiene menor porcentaje de fibras de colágeno lo que le confiere menor capacidad de resistir al estiramiento (Shultz y col., 2005; Shultz y col., 2008).

También encontramos estudios acerca de la relación entre la prevalencia del lesión de LCA y las diferentes fases del ciclo menstrual. El estudio de Wojtys, Huston, Lindenfeld, Hewett y Greenfield, (1998) relaciona la fase del ciclo mentrual de 40 atletas recientemente lesionadas de LCA con el momento de la lesión. Los resultados muestran diferencias signficativas entre las fases del ciclo menstrual, debido a que se produjeron un mayor número de lesiones en la fase ovulatoria (días 10 a 14) y menos en la fase folicular (días 1 a 9) (Wojtys y col., 1998). En referencia a esto, Shultz y col., (2005) estudiaron la laxitud del LCA en las diferentes fases del LCA, mostrando que la laxitud del LCA es hormona-dependiente, siendo mayor con el aumento de la concentración de estradiol, la cual se produce en la fase ovulatoria (Shultz y col., 2005). Estos resultados coinciden con la prevalencia de lesión mayor en una determinada fase del ciclo menstrual (fase ovulatoria) mostrada en el estudio descriptivo de Wojtys y col., (1998). Este factor debería ser tenido muy en cuenta a la hora de prescribir las cargas de entrenamiento específico dirigido a la prevención de esta lesión y la mejora de la fuerza en mujeres conociendo la fase del ciclo menstrual en la que se encuentra para obtener mejoras más eficientes.

\section{Programas de prevención y modificación de los factores de riesgo.}

En la tabla 3 podemos ver los estudios que han realizado una intervención mediante programas preventivos de lesiones de rodilla, en especial del LCA, tratando de modificar factores de riesgo anteriormente expuestos. Algunos de los programas expuestos proponen realizar los ejercicios de prevención durante el calentamiento mientras que otros lo realizan dentro de la parte para la mejora condicional. Los programas preventivos muestran partes comúnes que serán resumidas al final del texto como la reeducación de saltos y los cambios de dirección. 
Romero-Moraleda, B.; Cuéllar, A.; González, J.; Bastida, N.; Echarri, E.; Gallardo, J., y Paredes, V. (2017). Revisión de los factores de riesgo y los programas de prevención de la lesión del ligamento cruzado anterior en fútbol femenino: propuesta de prevención. RICYDE. Revista internacional de ciencias del deporte, 48(13), 117-138. https://doi.org/10.5232/ricyde2017.04803

Tabla 3 Resumen: estudios de intervención para la modificación o prevención de los factores de riesgo de LCA

\begin{tabular}{|c|c|c|c|c|c|}
\hline Autor & $\mathrm{N}$ & Intervención & $\begin{array}{l}\text { Tipo de } \\
\text { Estudio }\end{array}$ & Variables Dependientes & Resultado \\
\hline $\begin{array}{l}\text { Heidt, } \\
\text { Sweeterman, } \\
\text { Carlonas, Traub } \\
\text { y Tekulve, } \\
2000\end{array}$ & 300 Mujeres & $\begin{array}{c}\text { Grupo intervención }(\mathrm{n}=42) \\
\text { Grupo control }(\mathrm{n}=258) \\
7 \text { semanas de entrenamiento Frappier } \\
\text { Acceleration, basado en una combinación } \\
\text { de acondicionamiento cardiovascular, } \\
\text { pliometría, ejercicios con cuerda, } \\
\text { entrenamiento de fuerza, flexibilidad, } \\
\text { velocidad y agilidad, } 3 \text { sesiones por } \\
\text { semana. }\end{array}$ & $\begin{array}{l}\text { Ensayo clínico } \\
\text { aleatorizado }\end{array}$ & $\begin{array}{l}\text { Hoja de control de lesiones } \\
\text { Pre-test y Post-test habilidades } \\
\text { específicas del deporte: salto } \\
\text { vertical, salto de longitud, } \\
\text { carrera } 10 \text { yardas y agilidad } \\
\text { mediante ejercicios } \\
\text { pliométricos. }\end{array}$ & $\begin{array}{l}\downarrow \text { lesión LCA en grupo intervención } \\
(2,4 \%) \text { comparado con el grupo control } \\
(3,1 \%) \text {, a pesar de que no exista una } \\
\text { diferencia estadísticamente significativa } \\
\text { entre el grupo intervención y el grupo } \\
\text { control, se apunta a que el programa de } \\
\text { entrenamiento Frappier Acceleration } \\
\text { puede ser efectivo en la reducción de } \\
\text { lesiones en miembro inferior. }\end{array}$ \\
\hline $\begin{array}{l}\text { Irmischer, } \\
\text { Harris, Pfeiffer, } \\
\text { DeBeliso, } \\
\text { Adams y Shea, } \\
2004\end{array}$ & 28 Mujeres & $\begin{array}{c}\text { Grupo intervención }(\mathrm{n}=14) \\
\text { Grupo control }(\mathrm{n}=14) \\
9 \text { semanas de entrenamiento pliométrico } \\
\text { para la prevención de lesiones de } \\
\text { ligamento en la rodilla }(K L I P) \text {, basado en } \\
\text { ejercicios de baja intensidad de salto- } \\
\text { aterrizaje-salto, apropiadas para la } \\
\text { población activa general, se establecieron } \\
4 \text { fases, las } 3 \text { primeras con una duración de } \\
2 \text { semanas y la última con una duración de } \\
3 \text { semanas, } 2 \text { sesiones por semana, 20'. }\end{array}$ & $\begin{array}{l}\text { Estudio clínico } \\
\text { aleatorizado }\end{array}$ & $\begin{array}{c}\text { Test de fuerza para la } \\
\text { medición del pico de fuerza de } \\
\text { impacto vertical }\left(F_{p}\right) \text { y tasa de } \\
\text { desarrollo de fuerza }(R F D) \text { en } \\
\text { la amortiguación/aterrizaje de } \\
\text { salto. } \\
\text { Test salto vertical para la } \\
\text { medición de la altura } \\
\text { alcanzada. }\end{array}$ & $\begin{array}{c}\Downarrow \text { fuerza de impacto vertical }\left(F_{p}\right) \text { y } \downarrow \text { tasa } \\
\text { de desarrollo de fuerza }(R F D) \text { en la } \\
\text { amortiguación/aterrizaje del salto. Esto } \\
\text { conduciría a un menor riesgo de lesión en } \\
\text { la articulación de la rodilla durante el } \\
\text { aterrizaje. } \\
\uparrow \text { altura de salto vertical (CMJ brazos } \\
\text { libres) pero no es significativa } \\
\text { estadísticamente. }\end{array}$ \\
\hline $\begin{array}{l}\text { Mandelbaum y } \\
\text { col., } 2005\end{array}$ & $\begin{array}{c}5703 \\
\text { Mujeres }\end{array}$ & $\begin{array}{c}\mathrm{T}^{\mathrm{a}} 2000 \\
\text { Grupo intervención }(\mathrm{n}=1041) \\
\text { Grupo control }(\mathrm{n}=1905) \\
\mathrm{T}^{\mathrm{a}} 2001 \\
\text { Grupo intervención }(\mathrm{n}=844) \\
\text { Grupo control }(\mathrm{n}=1913) \\
2 \text { temporadas, entrenamiento } \\
\text { neuromuscular y propioceptivo }(P E P\end{array}$ & $\begin{array}{c}\text { Ensayo clínico } \\
\text { aletorizado }\end{array}$ & $\begin{array}{c}\text { Cuestionario sobre la lesión } \\
\text { específica de rodilla, historia y } \\
\text { duración. }\end{array}$ & $\begin{array}{c}\mathrm{T}^{\mathrm{a}} 2000 \downarrow 88 \% \text { lesión LCA respecto al } \\
\text { grupo control } \\
\mathrm{T}^{\mathrm{a}} 2001 \downarrow \\
\begin{array}{c}74 \% \text { lesión LCA en grupo } \\
\text { intervención }\end{array}\end{array}$ \\
\hline
\end{tabular}


Romero-Moraleda, B.; Cuéllar, A.; González, J.; Bastida, N.; Echarri, E.; Gallardo, J., y Paredes, V. (2017). Revisión de los factores de riesgo y los programas de prevención de la lesión del ligamento cruzado anterior en fútbol femenino: propuesta de prevención. RICYDE. Revista internacional de ciencias del deporte, 48(13), 117-138. https://doi.org/10.5232/ricyde2017.04803

\begin{tabular}{|c|c|c|c|c|c|}
\hline & & $\begin{array}{l}\text { program), basado en } 3 \text { actividades de } \\
\text { calentamiento, } 5 \text { técnicas de estiramiento, } \\
3 \text { ejercicios de fuerza, } 5 \text { actividades de } \\
\text { pliometría y } 3 \text { ejercicios de agilidad } \\
\text { específicos de fútbol. }\end{array}$ & & & \\
\hline $\begin{array}{l}\text { Pfeiffer, Shea, } \\
\text { Roberts, } \\
\text { Grandstrand y } \\
\text { Bond, } 2006\end{array}$ & $\begin{array}{c}1439 \\
\text { Mujeres }\end{array}$ & $\begin{array}{c}\text { Grupo intervención }(\mathrm{n}=577) \\
\text { Grupo control }(\mathrm{n}=862) \\
2 \text { años de programa pliometría, } 2 \text { sesiones } \\
\text { por semana } 20^{\prime} .(\text { KLIP Program })\end{array}$ & $\begin{array}{l}\text { Ensayo clínico } \\
\text { aleatorizado }\end{array}$ & $\begin{array}{l}\text { Control de lesión LCA vía } \\
\text { mail o teléfono }\end{array}$ & $\begin{array}{l}\text { El programa de pliometría durante } 20 \text { ’ no } \\
\text { reducía la incidencia lesional del LCA. }\end{array}$ \\
\hline $\begin{array}{l}\text { Pollard, } \\
\text { Sigwars, Ota, } \\
\text { Langford y } \\
\text { Powers, } 2006\end{array}$ & 18 Mujeres & $\begin{array}{c}\text { Grupo intervención }(\mathrm{n}=18) \\
\text { Programa de prevención de lesiones }(P E P \\
\text { program) 2-3 sesiones por semana. }\end{array}$ & $\begin{array}{c}\text { Estudio } \\
\text { prospectivo } \\
\text { experimental }\end{array}$ & $\begin{array}{l}\text { Medición de los ángulos de la } \\
\text { cadera y rodilla (abducción, } \\
\text { rotación interna, valgo, } \\
\text { flexión) en la fase de } \\
\text { desaceleración temprana en el } \\
\text { aterrizaje. }\end{array}$ & $\begin{array}{c}\Downarrow \text { rotación interna de la cadera y } \uparrow \text { de a } \\
\text { abducción de la cadera. No hubo } \\
\text { diferencias en el valgo de la rodilla ni en } \\
\text { los ángulos de flexión de la misma. Se } \\
\text { apunta a que durante una temporada de } \\
\text { entrenamiento en fútbol combinado con un } \\
\text { programa de prevención de lesiones, } \\
\text { alteraría eficazmente los movimientos de } \\
\text { tren inferior que predisponen a una lesión } \\
\text { del LCA. }\end{array}$ \\
\hline $\begin{array}{l}\text { Steffen, } \\
\text { Myklebust, } \\
\text { Olsen, Holme y } \\
\text { Bahr, 2008 }\end{array}$ & $\begin{array}{c}2020 \\
\text { Mujeres }\end{array}$ & $\begin{array}{c}\text { Grupo intervención }(\mathrm{n}=1073) \\
\text { Grupo control }(\mathrm{n}=947) \\
8 \text { meses de programa de entrenamiento de } \\
\text { core, fuerza en extremidad inferior, control } \\
\text { neuromuscular y agilidad denominado } \\
\text { "FIFA 11", 15' de calentamiento en cada } \\
\text { sesión de entrenamiento. }\end{array}$ & $\begin{array}{l}\text { Ensayo } \\
\text { aleatorizado } \\
\text { controlado }\end{array}$ & Registro de lesiones & $\begin{array}{l}\text { Tras la aplicación del programa de } \\
\text { entrenamiento " } 11 \text { " no se encontraron } \\
\text { diferencias significativas entre ambos } \\
\text { grupos debido a la baja participación. } \\
\text { Datos registrados de lesión del LCA fue de } \\
0,07 / 1000 \mathrm{~h} \text { en grupo intervención, frente } \\
0,8 / 1000 \mathrm{~h} \text { en grupo control. }\end{array}$ \\
\hline $\begin{array}{l}\text { Gilchrist y col., } \\
2008\end{array}$ & $\begin{array}{c}1435 \\
\text { Mujeres }\end{array}$ & $\begin{array}{c}\text { Grupo intervención }(\mathrm{n}=583) \\
\text { Grupo control }(\mathrm{n}=852) \\
12 \text { semanas de programa de entrenamiento } \\
\text { neuromuscular y propioceptivo, } 3 \text { veces } \\
\text { por semana (PEP program). }\end{array}$ & $\begin{array}{l}\text { Ensayo } \\
\text { aleatorizado } \\
\text { controlado }\end{array}$ & Hoja de control & $\begin{array}{l}\downarrow 70 \% \text { incidencia lesión LCA en grupo } \\
\text { intervención comparado con grupo control, } \\
\text { con un programa de entrenamiento } \\
\text { neuromuscular y propioceptivo }(P E P) \text {. }\end{array}$ \\
\hline LaBella y col., & 1492 & Grupo intervención M (n=737) & Ensayo & Hoja de control de lesiones, & Un calentamiento neuromuscular reduce \\
\hline
\end{tabular}


Romero-Moraleda, B.; Cuéllar, A.; González, J.; Bastida, N.; Echarri, E.; Gallardo, J., y Paredes, V. (2017). Revisión de los factores de riesgo y los programas de prevención de la lesión del ligamento cruzado anterior en fútbol femenino: propuesta de prevención. RICYDE. Revista internacional de ciencias del deporte, 48(13), 117-138. https://doi.org/10.5232/ricyde2017.04803

\begin{tabular}{|c|c|c|c|c|c|}
\hline 2011 & Mujeres & $\begin{array}{c}\text { Grupo control } \mathrm{M}(\mathrm{n}=755) \\
20 \text { ' calentamiento neuromuscular } \\
6 \text { semanas de KLIP program en } \\
\text { pretemporada }\end{array}$ & $\begin{array}{l}\text { aleatorizado } \\
\text { controlado }\end{array}$ & $\begin{array}{c}\text { entrevista a los jugadores } \\
\text { lesionados. }\end{array}$ & $\begin{array}{l}\text { significativamente lesiones LCA en } \\
\text { jugadoras de fútbol y baloncesto. } \\
0,10 / 1000 \mathrm{~h} \text { grupo de intervención } \\
\text { 0,46/1000h grupo control } \\
\text { Reducción del } 65 \% \text { de las lesiones. }\end{array}$ \\
\hline $\begin{array}{l}\text { Kiani, } \\
\text { Hellquist, } \\
\text { Ahlqvist, } \\
\text { Gedeborg y } \\
\text { Byberg, 2010 }\end{array}$ & $\begin{array}{c}1506 \\
\text { Mujeres }\end{array}$ & $\begin{array}{c}\text { Grupo intervención ( } \mathrm{n}=777 \text { jugadoras, } 48 \\
\text { equipos) } \\
\text { Grupo control ( } \mathrm{n}=729 \text { jugadoras, } 49 \\
\text { equipos). } \\
9 \text { meses de programa de entrenamiento } \\
\text { (HarmoKnee), basado en el control } \\
\text { corporal, activación muscular y desarrollo } \\
\text { de habilidades motoras específicas del } \\
\text { fútbol, compuesto por } 5 \text { partes } \\
\text { calentamiento, activación muscular, } \\
\text { equilibrio, fuerza y estabilidad de core, } 2 \\
\text { sesiones por semana desde Febrero a Abril } \\
\text { y posteriormente } 1 \text { sesión por semana. }\end{array}$ & $\begin{array}{c}\text { Ensayo } \\
\text { aleatorizado }\end{array}$ & $\begin{array}{c}\text { Exposición de horas de } \\
\text { entrenamiento, asistencia y } \\
\text { partido. } \\
\text { Información sobre el } \\
\text { cumplimiento del programa al } \\
\text { final de pretemporada y final } \\
\text { del periodo competitivo. } \\
\text { Reporte de la lesión al } \\
\text { investigador principal. }\end{array}$ & $\begin{array}{l}\text { La aplicación del programa (HarmoKnee) } \\
\downarrow \text { 90\% la incidencia lesional sin contacto } \\
\text { en grupo intervención, específicamente en } \\
\text { LCA de } 5 \text { lesiones documentadas ninguna } \\
\text { ocurrió en el grupo de intervención. }\end{array}$ \\
\hline $\begin{array}{c}\text { Hewett, } \\
\text { Lindenfeld, } \\
\text { Riccobene y } \\
\text { Noyes, } 1999\end{array}$ & $\begin{array}{c}829 \text { Mujeres } \\
434 \\
\text { Hombres }\end{array}$ & $\begin{array}{c}\text { Grupo intervención M }(\mathrm{n}=366) \\
\text { Grupo control } \mathrm{M}(\mathrm{n}=463) \\
\text { Grupo control } \mathrm{H}(\mathrm{n}=434) \\
6 \text { semanas de entrenamiento } \\
\text { neuromuscular, basado en trabajo } \\
\text { pliométrico, de flexibilidad y } \\
\text { entrenamiento de fuerza con peso libre, } \\
\text { con una duración de sesión de 60-90', } 3 \\
\text { veces por semana. }\end{array}$ & $\begin{array}{l}\text { Ensayo clínico } \\
\text { aleatorizado }\end{array}$ & $\begin{array}{c}\text { Cuestionario lesiones a lo } \\
\text { largo de su vida deportiva. } \\
\text { Entrega de informe de lesión } \\
\text { semanales. }\end{array}$ & $\begin{array}{c}\downarrow \text { lesiones de rodilla tras programa } \\
\text { específico de entrenamiento } \\
\text { neuromuscular (pliometría, flexibilidad y } \\
\text { entrenamiento de fuerza), en atletas de } \\
\text { sexo femenino. }\end{array}$ \\
\hline $\begin{array}{l}\text { Chimera, K.A. } \\
\text { Swanik, C.B. } \\
\text { Swanik y } \\
\text { Straub, } 2004\end{array}$ & 18 Mujeres & $\begin{array}{c}\text { Grupo intervención }(\mathrm{n}=9) \\
\text { Grupo control }(\mathrm{n}=9) \\
6 \text { semanas de entrenamiento pliométrico, } 2 \\
\text { sesiones por semana, 20'-30'. }\end{array}$ & $\begin{array}{c}\text { Ensayo clínico } \\
\text { aleatorizado }\end{array}$ & $\begin{array}{l}\text { Medición de la actividad } \\
\text { preparación y reactiva del } \\
\text { vasto medial, vasto externo, } \\
\text { isquiosurales, aductores y } \\
\text { abductores mediante }\end{array}$ & $\begin{array}{l}\boldsymbol{\uparrow} \text { activación de los aductores durante la } \\
\text { fase preparatoria, } \uparrow \text { coactivación } \\
\text { aductores y abductores y tendencia } \\
\text { reactiva del cuádriceps e isquiosurales. } \\
\text { Con ello se muestra una mejora en el } \\
\text { control y alineación del tren inferior al }\end{array}$ \\
\hline
\end{tabular}


Romero-Moraleda, B.; Cuéllar, A.; González, J.; Bastida, N.; Echarri, E.; Gallardo, J., y Paredes, V. (2017). Revisión de los factores de riesgo y los programas de prevención de la lesión del ligamento cruzado anterior en fútbol femenino: propuesta de prevención. RICYDE. Revista internacional de ciencias del deporte, 48(13), 117-138. https://doi.org/10.5232/ricyde2017.04803

\begin{tabular}{|c|c|c|c|c|c|}
\hline & & & & $\begin{array}{c}\text { electromiografía. } \\
\text { Altura de salto vertical (DJ) } \\
\text { Velocidad de sprint }\end{array}$ & $\begin{array}{c}\text { contactar con un terreno dinámico, es decir } \\
\text { hay una mejora de la estabilidad articular } \\
\text { pudiéndose reducir el riesgo de lesiones. } \\
\text { 个 altura de salto } 5,8 \% \text { grupo intervención } \\
\text { y } 2,0 \% \text { grupo control aunque no es } \\
\text { significativo. } \\
\text { 个 velocidad sprint pero no es significativo }\end{array}$ \\
\hline $\begin{array}{l}\text { Myer, Ford, } \\
\text { Brent y } \\
\text { Hewett, } 2006\end{array}$ & 19 Mujeres & $\begin{array}{c}\text { Grupo Pliometría }(\mathrm{n}=8) \\
\text { Grupo Equilibrio }(\mathrm{n}=11) \\
7 \text { semanas de entrenamiento pliométrico o } \\
\text { entrenamiento de equilibrio, } 3 \text { sesiones por } \\
\text { semana, 90'. }\end{array}$ & $\begin{array}{c}\text { Estudio } \\
\text { prospectivo } \\
\text { experimental }\end{array}$ & $\begin{array}{c}\text { Fuerza de extensión de rodilla } \\
\text { y flexión con dinamómetro } \\
\text { Salto sobre una pierna } \\
\text { (control de la desviación del } \\
\text { centro de presión) y equilibrio } \\
\text { Salto vertical } \\
\text { Fuerza isoinercial e } \\
\text { isocinética }\end{array}$ & $\begin{array}{l}\text { Ambos grupos demostraron efectos } \\
\text { diferentes en la técnica de } \\
\text { aterrizaje/amortiguación en el salto a una } \\
\text { pierna. } \\
\text { Diferencias significativas en la fuerza de } \\
\text { reacción en salto vertical siendo menor la } \\
\text { aplicada por el grupo de equilibrio. } \\
\text { Ambos grupos disminuyeron la desviación } \\
\text { del centro de presión. } \\
\text { Ambos grupos aumentaron la fuerza de } \\
\text { isquiotibiales y altura del salto vertical. }\end{array}$ \\
\hline $\begin{array}{l}\text { Myer, Ford, } \\
\text { Brent y } \\
\text { Hewett, } 2007\end{array}$ & 29 Mujeres & $\begin{array}{c}\text { Grupo intervención }(\mathrm{n}=18) \\
\text { Grupo control }(\mathrm{n}=11) \\
7 \text { semanas de entrenamiento } \\
\text { neuromuscular, } 3 \text { sesiones por semana }\end{array}$ & $\begin{array}{c}\text { Ensayo clínico } \\
\text { aleatorizado }\end{array}$ & $\begin{array}{l}\text { Medición de la cinemática y } \\
\text { cinética de la rodilla durante } \\
\text { el DVJ, obteniendo medición } \\
\text { de la abducción de la rodilla } \\
\text { mediante la dinámica inversa. }\end{array}$ & $\begin{array}{l}\text { El grupo de alto riesgo } \downarrow \text { el momento de } \\
\text { abducción en un } 13 \% \text { tras el programa de } \\
\text { entrenamiento, sin embargo el grupo de } \\
\text { bajo riesgo no modificó significativamente } \\
\text { el momento de abducción. } \\
\text { Las mujeres atletas incluidas dentro de alto } \\
\text { riesgo disminuyen la magnitud de riesgo a } \\
\text { sufrir una lesión del LCA tras el } \\
\text { entrenamiento neuromuscular, sin embargo } \\
\text { no llego a los niveles medios del grupo de } \\
\text { bajo riesgo, por lo que sería necesario un } \\
\text { mayor volumen de entrenamiento o } \\
\text { técnicas más específicas. }\end{array}$ \\
\hline
\end{tabular}


Romero-Moraleda, B.; Cuéllar, A.; González, J.; Bastida, N.; Echarri, E.; Gallardo, J., y Paredes, V. (2017). Revisión de los factores de riesgo y los programas de prevención de la lesión del ligamento cruzado anterior en fútbol femenino: propuesta de prevención. RICYDE. Revista internacional de ciencias del deporte, 48(13), 117-138. https://doi.org/10.5232/ricyde2017.04803

\begin{tabular}{|c|c|c|c|c|c|}
\hline $\begin{array}{l}\text { Chappell y } \\
\text { Limpisvasti, } \\
2008\end{array}$ & 30 Mujeres & $\begin{array}{c}\text { Grupo intervención }(\mathrm{n}=30) \\
6 \text { semanas de programa de entrenamiento } \\
\text { neuromuscular con fortalecimiento del } \\
\text { core y pliometría, } 6 \text { sesiones por semana, } \\
10-15\end{array}$ & $\begin{array}{c}\text { Estudio } \\
\text { prospectivo } \\
\text { experimental }\end{array}$ & $\begin{array}{c}\text { Medición de la fuerza de } \\
\text { reacción vertical del pie } \\
\text { derecho en plataforma de } \\
\text { fuerza. } \\
\text { Medición en la fase de apoyo } \\
\text { en el aterrizaje de los ángulos } \\
\text { iniciales de la flexión- } \\
\text { extensión, abducción- } \\
\text { aducción y rotación interna- } \\
\text { externa de la rodilla y cadera } \\
\text { y los ángulos máximos. } \\
\text { Medición del tiempo para } \\
\text { realizar } 20 \text { saltos a una pierna } \\
\text { y medición de la altura } \\
\text { máxima en salto vertical. }\end{array}$ & $\begin{array}{l}\downarrow \text { valgo dinámico de la rodilla durante la } \\
\text { fase de apoyo del salto de parada, en la } \\
\text { caída de salto no se observaron diferencias } \\
\text { ^ de los ángulos en la flexión de rodilla } \\
\text { inicial y máxima, durante el apoyo de la } \\
\text { caída del salto, pero no se observaron } \\
\text { diferencias respecto al de parada. } \\
\text { 个 rendimiento en salto vertical (DJ y Stop } \\
\text { Jump) y salto a una pierna tanto con la } \\
\text { derecha como con la izquierda. }\end{array}$ \\
\hline $\begin{array}{l}\text { Herman, } \\
\text { Weinhold, } \\
\text { Guskiewicz, } \\
\text { Garrett yu y } \\
\text { Padua, } 2008\end{array}$ & 66 Mujeres & $\begin{array}{l}\text { Grupo intervención }(\mathrm{n}=33) \\
\text { Grupo control }(\mathrm{n}=33) \\
9 \text { semanas de programa de entrenamiento } \\
\text { de fuerza para cuádriceps, isquiosurales, } \\
\text { glúteo medio y glúteo mayor, con bandas } \\
\text { de resistencia y balones, } 3 \text { sesiones por } \\
\text { semana. }\end{array}$ & $\begin{array}{l}\text { Estudio } \\
\text { controlado en } \\
\text { laboratorio }\end{array}$ & $\begin{array}{c}\text { Datos cinemáticos y cinéticos } \\
\text { en } 3 \text { dimensiones de rodilla y } \\
\text { cadera mientras realizaban } 3 \\
\text { saltos y parada. } \\
\text { Medición de la fuerza en } \\
\text { cuádriceps, isquiosurales, } \\
\text { glúteo mayor y glúteo medio } \\
\text { de la pierna dominante, } \\
\text { mediante un dinamómetro de } \\
\text { mano. } \\
\text { Medición de los ángulos de la } \\
\text { cadera y rodilla. }\end{array}$ & $\begin{array}{l}\text { 个 fuerza en el grupo de intervención. No } \\
\text { se observaron diferencias significativas en } \\
\text { la rodilla y cadera en la cinemática y } \\
\text { cinética entre ambos grupos. }\end{array}$ \\
\hline $\begin{array}{l}\text { Soligard y } \\
\text { col.,2010 }\end{array}$ & $\begin{array}{c}2275 \\
\text { Mujeres }\end{array}$ & $\begin{array}{l}\text { Grupo intervención }(\mathrm{n}=1055) \\
\text { Grupo control }(\mathrm{n}=1220) \\
\text { Desde finales de abril hasta mediados de } \\
\text { Octubre de } 2007 \text {, interrumpida por una de } \\
7 \text { semanas de verano descanso. También } \\
\text { fueron seguidos durante } 2 \text { meses de la }\end{array}$ & $\begin{array}{c}\text { Estudio } \\
\text { Prospectivo }\end{array}$ & $\begin{array}{l}\text { Minutos de exposición en } \\
\text { entrenamiento y partido. }\end{array}$ & $\begin{array}{c}\Downarrow 35 \% \text { riesgo jugadores con alto } \\
\text { cumplimiento programa de prevención. } \\
\downarrow 46 \% \text { riesgo jugadores cuyos } \\
\text { entrenadores aplicaban programa de } \\
\text { prevención de lesiones. }\end{array}$ \\
\hline
\end{tabular}


Romero-Moraleda, B.; Cuéllar, A.; González, J.; Bastida, N.; Echarri, E.; Gallardo, J., y Paredes, V. (2017). Revisión de los factores de riesgo y los programas de prevención de la lesión del ligamento cruzado anterior en fútbol femenino: propuesta de prevención. RICYDE. Revista internacional de ciencias del deporte, 48(13), 117-138. https://doi.org/10.5232/ricyde2017.04803

\begin{tabular}{|c|c|c|c|c|c|}
\hline & & $\begin{array}{c}\text { pretemporada (marzo-abril) } \\
2 \text { veces por semana }\end{array}$ & & & \\
\hline $\begin{array}{l}\text { Waldén, } \\
\text { Atroshi, } \\
\text { Magnusson, } \\
\text { Wagner y } \\
\text { Hägglund, 2012 }\end{array}$ & $\begin{array}{c}4564 \\
\text { Mujeres }\end{array}$ & $\begin{array}{c}\text { Grupo intervención }(\mathrm{n}=2479) \\
\text { Grupo control }(\mathrm{n}=2085) \\
15 \text {, de calentamiento neuromuscular, } 2 \\
\text { sesiones por semana }\end{array}$ & $\begin{array}{l}\text { Ensayo } \\
\text { aleatorizado } \\
\text { controlado }\end{array}$ & $\begin{array}{l}\text { Cuestionario de registro de } \\
\text { lesiones. }\end{array}$ & $\begin{array}{l}\text { Calentamiento neuromuscular } \downarrow 64 \% \text { tasa } \\
\text { de lesión de LCA en el grupo de } \\
\text { intervención en comparación con el grupo } \\
\text { control. }\end{array}$ \\
\hline $\begin{array}{c}\text { Steffen K, } \\
\text { Meeuwisse } \\
\text { WH, Romiti M, } \\
\text { Kang J, McKay } \\
\text { C, Bizzini M, et } \\
\text { al. } 2013\end{array}$ & $\begin{array}{l}385 \text { mujeres } \\
\text { entre } 13 \text { y } 19 \\
\text { años }\end{array}$ & $\begin{array}{l}\text { Grupo control }(n=135) \\
\text { Grupo menor adherencia }(n=121) \\
\text { Grupo mayor adherencia }(n=129)\end{array}$ & $\begin{array}{l}\text { Ensayo } \\
\text { aleatorizado } \\
\text { controlado }\end{array}$ & $\begin{array}{l}\text { Adherencia al programa. } \\
\text { Registro de lesiones. } \\
\text { Single leg balance. } \\
\text { Star Excursion Balance Test. } \\
\text { Single leg triple hop. }\end{array}$ & $\begin{array}{l}\text { La aplicación del programa FIFA } 11 \text { no } \\
\text { mostró diferencias en la incidencia de } \\
\text { lesión entre los tres grupos }\end{array}$ \\
\hline $\begin{array}{l}\text { Zebis, M. K., } \\
\text { Andersen, L. } \\
\text { L., Brandt, M., } \\
\text { Myklebust, G., } \\
\text { Bencke, J., } \\
\text { Lauridsen, H. } \\
\text { B., ... Aagaard, } \\
\text { P. (2015). }\end{array}$ & $\begin{array}{c}40 \text { mujeres } \\
\text { adolescentes } \\
(15-16 \text { años })\end{array}$ & $\begin{array}{l}\text { Grupo control }(\mathrm{n}=20) \\
\text { Grupo entrenamiento neuromuscular } \\
\qquad(\mathrm{n}=20) \\
\text { Programa } N M T \text {, calentamiento con } \\
\text { contenido preventivo antes del } \\
\text { entrenamiento } 3 \text { veces/semana durante } 12 \\
\text { semanas. Se incluía un incremento de la } \\
\text { dificultad de forma progresiva. }\end{array}$ & $\begin{array}{l}\text { Ensayo } \\
\text { aleatorizado } \\
\text { controlado }\end{array}$ & $\begin{array}{l}\text { Preactivación de Vasto } \\
\text { Lateral, Semitendinoso y } \\
\text { Bíceps Femoral en maniobra } \\
\text { de corte mediante EMG. } \\
\text { Ángulo de valgo en rodilla en } \\
\text { contacto inicial y valgo } \\
\text { máximo durante la maniobra, } \\
\text { análisis del movimiento 3D } \\
\text { Contracción isométrica } \\
\text { máxima de musculatura } \\
\text { isquiosural medición mediante } \\
\text { dinamómetro }\end{array}$ & $\begin{array}{c}\text { La aplicación de programa preventivo de } \\
12 \text { semanas de duración combinado con el } \\
\text { entrenamiento y competición en } \\
\text { deportistas adolescentes mejora las } \\
\text { estrategias de activación durante las } \\
\text { maniobras de corte. } \\
\downarrow \text { en la dominancia del cuádriceps } \\
\text { respecto de la musculatura isquiosural y } \uparrow \\
\text { la activación de la musculatura agonista } \\
\text { del LCA. }\end{array}$ \\
\hline
\end{tabular}


Romero-Moraleda, B.; Cuéllar, A.; González, J.; Bastida, N.; Echarri, E.; Gallardo, J., y Paredes, V. (2017). Revisión de los factores de riesgo y los programas de prevención de la lesión del ligamento cruzado anterior en fútbol femenino: propuesta de prevención. RICYDE. Revista internacional de ciencias del deporte, 48(13), $117-138$. https://doi.org/10.5232/ricyde2017.04803

En los estudios de intervención cuyo objetivo fue cambiar los factores de riesgo modificables en la lesión del LCA incidieron en la medición de la activación de la musculatura periférica (Chimera, Swanik, Swanik y Straub, 2004; Herman y col., 2008; Lephart y col., 2005), los grados de flexión de cadera-rodilla-tobillo en la amortiguación de los saltos (Chappell y Limpisvasti, 2008; Lephart y col., 2005), los momentos de rotación interna y valgo de rodilla y abducción de cadera (Chappell y Limpisvasti, 2008; Chimera y col., 2004; Lephart y col., 2005; Myer, Ford, Brent y Hewett, 2006; Pollard, Sigward, Ota, Langford y Powers, 2006), las fuerzas de impacto vertical (Irmischer y col., 2004; Lephart y col., 2005), la anteriorización de la tibia en el salto (Herman y col., 2008) y la mejora del equilibrio y control del tronco (postura) (Paterno, Myer, Ford y Hewett, 2004).

En cuanto a la modificación de la preactivación y activación de la musculatura periférica de rodilla y tobillo, una intervención de entre 6 y 8 semanas consiguió una mayor activación temprana en la fase preparatoria del salto de la musculatura aductora de cadera (Chimera y col., 2004; Lephart y col., 2005). También se mostró una mayor coactivación de isquiosurales, cuádriceps, aductores y abductores en la amortiguación del salto lo que refiere una mayor estabilización de la rodilla. El trabajo de Lephar y colaboradores también encontró mayor activación del glúteo medio y mayor en la recepción del salto (Lephart y col., 2005). Es importante incluir en los programas de prevención este tipo de ejercicios para conseguir la preactivación y coactivación de la musculatura de rodilla y cadera que interviene tanto en los saltos como en los cambios de dirección, protegiendo así del mecanismo lesional del LCA.

En cuanto a los estudios que realizan una reeducación del salto, han demostrado aumentar los grados de flexión de cadera y rodilla y rotación externa de rodilla, así como una mayor preactivación del glúteo medio y fuerza reactiva (Chappell y Limpisvasti, 2008; Lephart y col., 2005). En referencia a esta mayor activación del glúteo medio, el trabajo de Myer y col. (2007) justifica que la disminución significativa de un $13 \%$ del valgo de rodilla puede ser debido a ello (Myer, Ford, Brent y Hewett, 2007). La mayor activación de la musculatura aductora de cadera puede ser la causante de una mejora en la abducción de cadera mostrada en el trabajo de Myer y colaboradores y por tanto de un mejor mantenimiento de la postura (Myer y col., 2007). Dentro de este trabajo, es importante destacar que se clasificó a la muestra en "bajo riesgo" a aquellos que realizaban un valgo de rodilla por debajo de los 25,5 $\mathrm{Nm}$ y de "alto riesgo" a los que estaban por encima. Por ello, los resultados fueron más significativos en la muestra de "alto riesgo" que fue intervenida. Esto destaca la importancia de realizar una evaluación pre de los factores de riesgo para incidir en aquellos que son modificibles de forma más específica.

En cuanto a las fuerzas de reacción, el trabajo de Lephart y col. (2005) no mostró una disminución significativa tras la intervención, mientras que Irmischer y col. (2004) si consiguieron una disminución significativa de las fuerzas de reacción contra el suelo. Las posibles diferencias en estos resultados pueden deberse debido a que el entrenamiento realizado por Irmischer y col. (2004). es de mayor duración y con entrenamiento pliométrico desde la primera sesión, mientras que en la publicación Lephart y col. (2005) sólo se realizaron 4 semanas de entrenamiento pliométrico.

Además de las intervenciones dirigidas a la disminución del valgo de rodilla y rotación interna, junto a la mejora en la amortiguación en el plano sagital y en las fuerzas de reacción contra el suelo, el trabajo de Paterno y col. (2004) hace especial hincapié en la mejora de la estabilidad y el control postural para la prevención de lesión de LCA. En esta línea, Zazulak y col. (2007) expone que la desviación lateral del tronco en el plano frontal puede ser un buen predictor del riesgo de lesión del LCA, es por ello que además de los cambios cinemáticos en 
Romero-Moraleda, B.; Cuéllar, A.; González, J.; Bastida, N.; Echarri, E.; Gallardo, J., y Paredes, V. (2017). Revisión de los factores de riesgo y los programas de prevención de la lesión del ligamento cruzado anterior en fútbol femenino: propuesta de prevención. RICYDE. Revista internacional de ciencias del deporte, 48(13), $117-138$. https://doi.org/10.5232/ricyde2017.04803

el plano sagital y frontal de miembros inferiores es necesario trabajar la estabilidad postural del tronco.

Por otro lado encontramos estudios donde han tratado de comprobar la eficacia que tiene la aplicación de varios programas diseñados por expertos para la prevención de lesiones de rodilla. El PEP Program (Prevent Injury and Enhance Performance Program) es un programa sencillo que no requiere material ni mucho tiempo de aplicación en la sesión, debido a que puede servir como calentamiento al inicio. Dos estudios prospectivos donde realizaron la aplicación del programa durante 12 semanas y durante y dos temporadas, respectivamente, obtuvieron una disminución significativa de la incidencia de lesión del LCA con respecto al grupo control (Gilchrist y col., 2008; Mandelbaum y col., 2005). Gilchirist y col. (2008) disminuyeron la incidencia lesional un 70\% mientras que Mandelbaum y col. (2005) obtuvo una reducción del $88 \%$ en la primera temporada de aplicación del programa y un $74 \%$ de lesión del LCA en el segundo año.

Otro de los programas utilizado para la prevención de lesiones general es el FIFA $11+$, el cual incluye ejercicios de carrera de diferentes tipos con ejercicios de fuerza, pliometría y equilibrio. Soligard y col. (2010) mostraron una disminución de entre el 35 y el 46\% según la adherencia al programa (Soligard y col., 2010). Steffen y col. (2008) no encontraron diferencia significativas en la disminución lesional entre el grupo de intervención con el FIFA $11+$ y el grupo control (Steffen, Myklebust, Olsen, Holme y Bahr, 2008). La controversia de resultados entre estos dos estudios puede ser explicada por la baja adherencia final en el trabajo de Steffen y col., la cual fue de un $52 \%$. En esta línea, el trabajo de Steffen y col. (2013) llevado a cabo en jugadoras canadienses obtuvo una disminución del $57 \%$ de la frecuencia lesional en el grupo de alta adherencia frente al de baja adherencia (Steffen y col., 2013).

El estudio de Kiani, Hellquist, Ahlqvist, Gedeborg y Byberg, (2010) trató de comprobar la eficacia del programa HarmoKnee, consistente en ejercicio de control corporal, activación muscular, fuerza, equilibrio y trabajo del core. Tras 9 meses de intervención en 49 equipos de jugadoras entre 13 y 19 años comprobaron un 90\% menos de incidencia lesional que en el grupo control, reportando que de las 5 lesiones del LCA, todas ocurrieron en el grupo control (Kiani y col., 2010).

Otro de los programas preventivos evaluados fue el KLIP Program (Knee Ligament Injury Prevention) basado en un programa neuromuscular que incluía ejercicios de fortalecimiento, pliometría, equilibrio y agilidad incidiendo en la flexión de miembros inferiores y en una correcta amortiguación del salto evitando el valgo de rodilla. El trabajo de LaBella y col. (2011) mostró una reducción del 65\% de las lesiones de no contacto tras la realización del KLIP Program durante 6 semanas en pretemporada (LaBella y col.,2011). Sin embargo, el estudio de Pfeiffer y col. (2006) donde aplicaron el KLIP Program no consiguieron dismiuir la incidencia lesional del LCA en el grupo de intervención comparado con el grupo control (Pfeiffer, Shea, Roberts, Grandstrand y Bond, 2006).

Se puede destacar que los programas de prevención analizados muestran efectividad realizándolos un mínimo de 2 veces/semana, entre 20-30 min. La duración media de los programas analizados son de 4-9 semanas, con el fin de mostrar los resultados a nivel estadístico, sin embargo la aplicación de estos programas debe ser durante toda la temporada. A pesar de todos los programas de prevención (PEP, FIFA 11+, KLIP, HarmoKnee) lo ideal sería realizar programas de prevención individualizado a cada jugadora, adaptado a sus características y sus deficiencias para así posteriormente elaborar un plan de prevención óptimo, que le permita reducir al mínimo la probabilidad de padecer una lesión. 
Romero-Moraleda, B.; Cuéllar, A.; González, J.; Bastida, N.; Echarri, E.; Gallardo, J., y Paredes, V. (2017). Revisión de los factores de riesgo y los programas de prevención de la lesión del ligamento cruzado anterior en fútbol femenino: propuesta de prevención. RICYDE. Revista internacional de ciencias del deporte, 48(13), 117-138. https://doi.org/10.5232/ricyde2017.04803

\section{Conclusión}

Los factores de riesgo de la lesión de ligamento cruzado anterior en mujeres futbolistas se centran como factores modificables en: mayor valgo de rodilla, menor de grado de flexión de cadera, rodilla, tobillo en la amortiguación del salto, mayor rotación interna de cadera y de las fuerzas de impacto lo que supone una mayor traslación anterior de la tibia y riesgo de lesión del LCA. En cuanto a los factores no modificables encontramos: mayor ángulo Q, el valgo de rodilla estático, el genu recurvatum, el pie pronador y la estructura del LCA de la mujer que es más pequeño en longitud y de menor sección transversal que la del hombre y con menor porcentaje de fibras colágeno.

Las intervenciones enfocadas a la modificación de los factores de riesgo modificables consiguen atenuar el riesgo de lesión. Los programas de prevención mostraron ser eficaces en la disminución de la frecuencia lesional del LCA entre un 36\% y 80\%.

Los programas de prevención analizados incluyen protocolos de calentamiento con ejercicios para la mejora de la fuerza neuromucular, estiramientos, pliometría, equilibrio, core y corrección de la técnica de amortiguación del salto y los cambios de dirección.

En base a la evidencia científica mostrada resulta determinante considerar el trabajo de estos factores teniendo en cuenta la fase del ciclo menstrual donde se encuentre la jugadora, considerando los cambios de laxitud ligamentosa que se producen. En esta misma línea se destaca la necesidad de planificar las cargas del entrenamiento de fuerza teniendo en cuenta también las fases del ciclo menstrual para obtener mejoras neuromusculares más eficientes.

\section{Aplicación práctica:}

En la tabla 4 se presentan resumidos los ejercicios de los diferentes programas de prevención que han mostrado tener eficacia en la reducción de lesiones:

Tabla 4. Resumen de los ejercicios utilizados en los diferentes programas de prevención.

\begin{tabular}{|c|c|c|}
\hline & DURACIÓN & EJERCICIOS \\
\hline Calentamiento & $10 / 15$ minutos & $\begin{array}{l}\text { 1. Trote suave } \\
\text { 2. Carrera hacia atrás } \\
\text { 3. Carrera cogiendo un objeto } \\
\text { Incidir en la correcta técnica de carrera y el } \\
\text { aterrizaje tras salto y apoyo monopodal. }\end{array}$ \\
\hline Fortalecimiento & $\begin{array}{l}2 \text { × } 20 \text { rep. } \\
20 \text { rep. } \\
30 \text { rep. (cada pierna) }\end{array}$ & $\begin{array}{ll}\text { 1. Zancadas } \\
\text { 2. Flexiones rusas } 20 \text { repeticiones } \\
\text { 3. Single Toe Raises: en apoyo monopodal } \\
\text { ponerte de puntillas }\end{array}$ \\
\hline $\begin{array}{c}\text { Ejercicios de fuerza } \\
\text { excéntrica }\end{array}$ & $\begin{array}{l}3 \times 5 \text { rep. } \\
2 \times 12 / 15 \text { rep. } \\
2 \times 12 / 15 \text { rep. }\end{array}$ & $\begin{array}{ll}\text { 1. } & \text { Cinturón ruso } \\
\text { 2. } & \text { Ejercicios } L E G C U R L \\
\text { 3. } & \text { Ejercicios } L E G \text { PRESS }\end{array}$ \\
\hline Pliometría & $\begin{array}{l}20 \text { repeticiones }(2 \\
\text { piernas y con cada } \\
\text { pierna) }\end{array}$ & $\begin{array}{l}\text { 1. Saltos de lado a lado } \\
\text { 2. Saltos delante-atrás } \\
\text { 3. Saltos delante-atrás con una pierna } \\
\text { 4. Saltos verticales } \\
\text { 5. Zancada con salto }\end{array}$ \\
\hline
\end{tabular}


Romero-Moraleda, B.; Cuéllar, A.; González, J.; Bastida, N.; Echarri, E.; Gallardo, J., y Paredes, V. (2017). Revisión de los factores de riesgo y los programas de prevención de la lesión del ligamento cruzado anterior en fútbol femenino: propuesta de prevención. RICYDE. Revista internacional de ciencias del deporte, 48(13), $117-138$. https://doi.org/10.5232/ricyde2017.04803

\begin{tabular}{|c|c|c|}
\hline Agilidad & $20 \mathrm{rep} / 2$ series & $\begin{array}{l}\text { 1. Carrera delante-detrás } \\
\text { 2. Carrera en diagonal } \\
\text { 3. Carrera saltando + cambio de dirección }\end{array}$ \\
\hline Propiocepción & 2 series/ $30 \mathrm{seg}$ & $\begin{array}{l}\text { 1. Sentado en un fit- ball con los pies } \\
\text { apoyados en el suelo y un compañero nos } \\
\text { intenta desequilibrar } \\
\text { 2. Igual el anterior pero monopodal. Para } \\
\text { aumentar la dificultad se le lanzará una } \\
\text { pelota. } \\
\text { 3. En apoyo monopodal sobre el suelo: 1) } \\
\text { Base inestable; 2) Moviendo la extremidad } \\
\text { que no apoya; 3) Ojos cerrados; 4) } \\
\text { Lanzándole un balón a varias alturas } \\
\text { En apoyo monopodal sobre el suelo y con } \\
\text { las manos sobre las caderas, realiza flexo- } \\
\text { extensiones de rodilla (sentadillas) }\end{array}$ \\
\hline Core & $\begin{array}{l}2 \times 20 \text { rep. } \\
30^{\prime \prime}-1 \text { minuto }\end{array}$ & $\begin{array}{l}\text { 1. Nordic Hamstring lower (Nordic Ham } \\
\text { Curl) } \\
\text { 2. Abdominales transverso "plancha" con } \\
\text { codos apoyados. } \\
\text { 3. Abdominales transverso "plancha lateral" } \\
\text { un lado con codo apoyado }\end{array}$ \\
\hline $\begin{array}{c}\text { Análisis biomecánico de la } \\
\text { rodilla }\end{array}$ & \multicolumn{2}{|c|}{$\begin{array}{l}\text { Concienciación de una posición correcta de la rodilla en todos los ejercicios } \\
\text { anteriores. } \\
\text { Enseñar técnica de carrera y técnica de amortiguación tras salto. } \\
\text { Incluir trabajo para la mejora del valgo dinámico de rodilla en la flexión de la } \\
\text { misma en cadena cinética cerrada (amortiguación monopodal). Este gesto es } \\
\text { medible a través del Test Single Leg Squat. } \\
\text { En cuanto a la biomecánica de amortiguación remitimos al lector al trabajo de } \\
\text { Wesley y col. (2015) para la descripción de la misma y el conocimiento de los } \\
\text { cambios que pudieran producirse derivados de la fatiga. }\end{array}$} \\
\hline
\end{tabular}

\section{Referencias}

Acevedo, R. J.; Rivera-Vega, A.; Miranda, G., \& Micheo, W. (2014). Anterior cruciate ligament injury: identification of risk factors and prevention strategies. Current sports medicine reports, 13(3), 186-191.

https://doi.org/10.1249/JSR.0000000000000053

Beynnon, B. D.; Vacek, P. M.; Newell, M. K.; Tourville, T. W.; Smith, H. C.; Shultz, S. J., ... Johnson, R. J. (2014). The effects of level of competition, sport, and sex on the incidence of first-time noncontact anterior cruciate ligament injury. The American journal of sports medicine, 42(8), 1806-1812.

https://doi.org/10.1177/0363546514540862

Chappell, J. D., \& Limpisvasti, O. (2008). Effect of a neuromuscular training program on the kinetics and kinematics of jumping tasks. The American journal of sports medicine, 36(6), 1081-1086.

https://doi.org/10.1177/0363546508314425 
Romero-Moraleda, B.; Cuéllar, A.; González, J.; Bastida, N.; Echarri, E.; Gallardo, J., y Paredes, V. (2017). Revisión de los factores de riesgo y los programas de prevención de la lesión del ligamento cruzado anterior en fútbol femenino: propuesta de prevención. RICYDE. Revista internacional de ciencias del deporte, 48(13), $117-138$. https://doi.org/10.5232/ricyde2017.04803

Chimera, N. J.; Swanik, K. A.; Swanik, C. B., \& Straub, S. J. (2004). Effects of plyometric training on muscle-activation strategies and performance in female athletes. Journal of athletic training, 39(1), 24-31.

Elias, A., \& Mizner, R. (2014). Mechanism of injury affects neuromuscular patterning of hamstrings and quadriceps following anterior cruciate ligament reconstruction. Paper presented at the International Journal of Exercise Science: Conference Proceedings.

Faunø, P., \& Wulff, J. B. (2006). Mechanism of anterior cruciate ligament injuries in soccer. International journal of sports medicine, 27(1), 75-79. https://doi.org/10.1055/s-2005-837485

Gilchrist, J.; Mandelbaum, B. R.; Melancon, H.; Ryan, G. W.; Silvers, H. J.; Griffin, L. Y.; ... Dvorak, J. (2008). A randomized controlled trial to prevent noncontact anterior cruciate ligament injury in female collegiate soccer players. The American journal of sports medicine, 36(8), 1476-1483.

https://doi.org/10.1177/0363546508318188

Griffin, L. Y.; Albohm, M. J.; Arendt, E. A.; Bahr, R.; Beynnon, B. D.; DeMaio, M.; . . . Hannafin, J. A. (2006). Understanding and Preventing Noncontact Anterior Cruciate Ligament Injuries A Review of the Hunt Valley II Meeting, January 2005. The American journal of sports medicine, 34(9), 1512-1532. https://doi.org/10.1177/0363546506286866

Grimm, K. y Kirkendall, D. (2007). Salud y buena condición física de las futbolistas.: $1^{\text {a }}$ ed. FIFA.

Hägglund M, Waldén M.(2016) Risk factors for acute knee injury in female youth football. Knee surgery, sports traumatology, arthroscopy. 24(3), 737-46. https://doi.org/10.1007/s00167-015-3922-z

Herman, D. C.; Weinhold, P. S.; Guskiewicz, K. M.; Garrett, W. E.; Yu, B., \& Padua, D. A. (2008). The effects of strength training on the lower extremity biomechanics of female recreational athletes during a stop-jump task. The American journal of sports medicine, 36(4), 733-740. https://doi.org/10.1177/0363546507311602

Hewett, T.; Ford, K.; Hoogenboom, B., \& Myer, G. (2010). Understanding and preventing ACL injuries: current biomechanical and epidemiologic considerations - update 2010. North American Journal of Sports Physical Therapy, 5(4), 234.

Irmischer, B. S.; Harris, C.; Pfeiffer, R. P.; DeBeliso, M. A.; Adams, K. J., \& Shea, K. G. (2004). Effects of a knee ligament injury prevention exercise program on impact forces in women. The Journal of Strength y Conditioning Research, 18(4), 703-707. https://doi.org/10.1519/00124278-200411000-00003

Joseph, A. M.; Collins, C. L.; Henke, N. M.; Yard, E. E.; Fields, S. K., \& Comstock, R. D. (2012). A multisport epidemiologic comparison of anterior cruciate ligament injuries in high school athletics. Journal of athletic training, 48(6), 810-817. https://doi.org/10.4085/1062-6050-48.6.03

Kiani, A.; Hellquist, E.; Ahlqvist, K.; Gedeborg, R., \& Byberg, L. (2010). Prevention of soccer-related knee injuries in teenaged girls. Archives of internal medicine, 170(1), 43-49. https://doi.org/10.1001/archinternmed.2009.289

Kwan, M. K.; Lin, T. H., \& Woo, S. L. (1993). On the viscoelastic properties of the anteromedial bundle of the anterior cruciate ligament. Journal of biomechanics, 26(4), 447-452.

https://doi.org/10.1016/0021-9290(93)90008-3 
Romero-Moraleda, B.; Cuéllar, A.; González, J.; Bastida, N.; Echarri, E.; Gallardo, J., y Paredes, V. (2017). Revisión de los factores de riesgo y los programas de prevención de la lesión del ligamento cruzado anterior en fútbol femenino: propuesta de prevención. RICYDE. Revista internacional de ciencias del deporte, 48(13), $117-138$. https://doi.org/10.5232/ricyde2017.04803

LaBella, C. R.; Huxford, M. R.; Grissom, J.; Kim, K.-Y.; Peng, J., \& Christoffel, K. K. (2011). Effect of neuromuscular warm-up on injuries in female soccer and basketball athletes in urban public high schools: cluster randomized controlled trial. Archives of pediatrics $y$ adolescent medicine, 165(11), 1033-1040.

https://doi.org/10.1001/archpediatrics.2011.168

Leathers, M. P.; Merz, A.; Wong, J.; Scott, T.; Wang, J. C. y Hame, S. L. (2015). Trends and Demographics in Anterior Cruciate Ligament Reconstruction in the United States. $J$ Knee Surg, 28(5), 390-394.

https://doi.org/10.1055/s-0035-1544193

Lephart, S. M.; Abt, J.; Ferris, C.; Sell, T.; Nagai, T.; Myers, J. B., \& Irrgang, J. (2005). Neuromuscular and biomechanical characteristic changes in high school athletes: a plyometric versus basic resistance program. British journal of sports medicine, 39(12), 932-938.

https://doi.org/10.1136/bjsm.2005.019083

Lohmander, L.; Östenberg, A.; Englund, M., \& Roos, H. (2004). High prevalence of knee osteoarthritis, pain, and functional limitations in female soccer players twelve years after anterior cruciate ligament injury. Arthritis y Rheumatism, 50(10), 3145-3152. https://doi.org/10.1002/art.20589

MacLeod, T. D.; Snyder-Mackler, L., \& Buchanan, T. S. (2014). Differences in neuromuscular control and quadriceps morphology between potential copers and noncopers following anterior cruciate ligament injury. journal of orthopaedic $y$ sports physical therapy, 44(2), 76-84.

https://doi.org/10.2519/jospt.2014.4876

Mandelbaum, B. R.; Silvers, H. J.; Watanabe, D. S.; Knarr, J. F.; Thomas, S. D.; Griffin, L. Y.; . . . Garrett, W. (2005). Effectiveness of a Neuromuscular and Proprioceptive Training Program in Preventing Anterior Cruciate Ligament Injuries in Female Athletes 2-Year Follow-up. The American journal of sports medicine, 33(7), 1003-1010. https://doi.org/10.1177/0363546504272261

McCall, A.; Carling, C.; Nedelec, M.; Davison, M.; Le Gall, F.; Berthoin, S. \& Dupont, G. (2014). Risk factors, testing and preventative strategies for non-contact injuries in professional football: current perceptions and practices of 44 teams from various premier leagues. Br J Sports Med, 48(18), 1352-1357.

https://doi.org/10.1136/bjsports-2014-093439

Myer, G. D.; Ford, K. R.; Brent, J. L., \& Hewett, T. E. (2006). The effects of plyometric vs. dynamic stabilization and balance training on power, balance, and landing force in female athletes. The Journal of Strength y Conditioning Research, 20(2), 345-353. https://doi.org/10.1519/r-17955.1

Myer, G. D.; Ford, K. R.; Brent, J. L., \& Hewett, T. E. (2007). Differential neuromuscular training effects onACL injury risk factors in" high-risk" versus" low-risk" athletes. BMC musculoskeletal disorders, 8(1), 1. https://doi.org/10.1186/1471-2474-8-39

Myer, G. D.; Sugimoto, D.; Thomas, S. \& Hewett, T. E. (2013). The influence of age on the effectiveness of neuromuscular training to reduce anterior cruciate ligament injury in female athletes: a meta-analysis. Am J Sports Med, 41(1), 203-215.

https://doi.org/10.1177/0363546512460637

Panjabi, M. M. (1992). The stabilizing system of the spine. Part I. Function, dysfunction, adaptation, and enhancement. Journal of spinal disorders $y$ techniques, 5(4), 383389.

https://doi.org/10.1097/00002517-199212000-00001 
Romero-Moraleda, B.; Cuéllar, A.; González, J.; Bastida, N.; Echarri, E.; Gallardo, J., y Paredes, V. (2017). Revisión de los factores de riesgo y los programas de prevención de la lesión del ligamento cruzado anterior en fútbol femenino: propuesta de prevención. RICYDE. Revista internacional de ciencias del deporte, 48(13), $117-138$. https://doi.org/10.5232/ricyde2017.04803

Paterno, M. V.; Myer, G. D.; Ford, K. R., \& Hewett, T. E. (2004). Neuromuscular training improves single-limb stability in young female athletes. journal of orthopaedic $y$ sports physical therapy, 34(6), 305-316.

https://doi.org/10.2519/jospt.2004.34.6.305

Pauzenberger, L.; Syré, S., \& Schurz, M. (2013). "Ligamentization" in Hamstring Tendon Grafts After Anterior Cruciate Ligament Reconstruction: A Systematic Review of the Literature and a Glimpse Into the Future. Arthroscopy: The Journal of Arthroscopic $y$ Related Surgery, 29(10), 1712-1721.

https://doi.org/10.1016/j.arthro.2013.05.009

Pfeiffer, R. P.; Shea, K. G.; Roberts, D.; Grandstrand, S., \& Bond, L. (2006). Lack of effect of a knee ligament injury prevention program on the incidence of noncontact anterior cruciate ligament injury. The Journal of Bone $y$ Joint Surgery, 88(8), 17691774.

https://doi.org/10.2106/00004623-200608000-00012

Pollard, C. D.; Sigward, S. M.; Ota, S.; Langford, K., \& Powers, C. M. (2006). The influence of in-season injury prevention training on lower-extremity kinematics during landing in female soccer players. Clinical Journal of Sport Medicine, 16(3), 223-227. https://doi.org/10.1097/00042752-200605000-00006

Quatman, C. E., \& Hewett, T. E. (2009). The anterior cruciate ligament injury controversy: is "valgus collapse" a sex-specific mechanism? British journal of sports medicine, 43(5), 328-335.

https://doi.org/10.1136/bjsm.2009.059139

Ralles, S.; Agel, J.; Obermeier, M. \& Tompkins, M. (2015). Incidence of Secondary Intraarticular Injuries With Time to Anterior Cruciate Ligament Reconstruction. Am J Sports Med, 43(6), 1373-1379.

https://doi.org/10.1177/0363546515574061

Schutte, M. J.; Dabezies, E.; Zimny, M., \& Happel, L. (1987). Neural anatomy of the human anterior cruciate ligament. J Bone Joint Surg Am, 69(2), 243-247.

https://doi.org/10.2106/00004623-198769020-00011

Shultz, S.; Sander, T.; Kirk, S., \& Perrin, D. (2005). Sex differences in knee joint laxity change across the female menstrual cycle. The Journal of sports medicine and physical fitness, 45(4), 594.

Shultz, S. J.; Nguyen, A.-D., \& Schmitz, R. J. (2008). Differences in lower extremity anatomical and postural characteristics in males and females between maturation groups. journal of orthopaedic $y$ sports physical therapy, 38(3), 137-149. https://doi.org/10.2519/jospt.2008.2645

Sigward, S.; Pollard, C., \& Powers, C. (2012). The influence of sex and maturation on landing biomechanics: implications for anterior cruciate ligament injury. Scandinavian journal of medicine $y$ science in sports, 22(4), 502-509. https://doi.org/10.1111/j.1600-0838.2010.01254.x

Smith, H. C.; Vacek, P.; Johnson, R. J.; Slauterbeck, J. R.; Hashemi, J.; Shultz, S., \& Beynnon, B. D. (2012). Risk factors for anterior cruciate ligament injury a review of the literature-Part 1: neuromuscular and anatomic risk. Sports Health: $A$ Multidisciplinary Approach, 4(1), 69-78.

https://doi.org/10.1177/1941738111428281

Söderman K, Pietilä T, Alfredson H, Werner S. )2002) Anterior cruciate ligament injuries in young females playing soccer at senior levels. Scandinavian journal of medicine \& science in sports, 12(2), 65-8.

https://doi.org/10.1034/j.1600-0838.2002.120201.x 
Romero-Moraleda, B.; Cuéllar, A.; González, J.; Bastida, N.; Echarri, E.; Gallardo, J., y Paredes, V. (2017). Revisión de los factores de riesgo y los programas de prevención de la lesión del ligamento cruzado anterior en fútbol femenino: propuesta de prevención. RICYDE. Revista internacional de ciencias del deporte, 48(13), $117-138$. https://doi.org/10.5232/ricyde2017.04803

Soligard, T.; Nilstad, A.; Steffen, K.; Myklebust, G.; Holme, I.; Dvorak, J.; . . A Andersen, T. E. (2010). Compliance with a comprehensive warm-up programme to prevent injuries in youth football. British journal of sports medicine, 44(11), 787-793. https://doi.org/10.1136/bjsm.2009.070672

Steffen, K.; Meeuwisse, W. H.; Romiti, M.; Kang, J.; McKay, C.; Bizzini, M.; . . . Emery, C. A. (2013). Evaluation of how different implementation strategies of an injury prevention programme (FIFA $11+$ ) impact team adherence and injury risk in Canadian female youth football players: a cluster-randomised trial. $\mathrm{Br} J$ Sports Med, 47(8), 480487.

https://doi.org/10.1136/bjsports-2012-091887

Steffen, K.; Myklebust, G.; Olsen, O. E.; Holme, I., \& Bahr, R. (2008). Preventing injuries in female youth football-a cluster-randomized controlled trial. Scandinavian journal of medicine $y$ science in sports, 18(5), 605-614.

https://doi.org/10.1111/j.1600-0838.2007.00703.x

Stevenson, J. H.; Beattie, C. S.; Schwartz, J. B. \& Busconi, B. D. (2015). Assessing the effectiveness of neuromuscular training programs in reducing the incidence of anterior cruciate ligament injuries in female athletes: a systematic review. Am J Sports Med, 43(2), 482-490.

https://doi.org/10.1177/0363546514523388

Stracciolini, A.; Stein, C. J.; Zurakowski, D.; Meehan, W. P.; Myer, G. D. \& Micheli, L. J. (2015). Anterior cruciate ligament injuries in pediatric athletes presenting to sports medicine clinic: a comparison of males and females through growth and development. Sports Health, 7(2), 130-136.

https://doi.org/10.1177/1941738114554768

Sugimoto, D.; Myer, G. D.; Foss, K. D. B., \& Hewett, T. E. (2014). Dosage effects of neuromuscular training intervention to reduce anterior cruciate ligament injuries in female athletes: meta-and sub-group analyses. Sports Medicine, 44(4), 551-562. https://doi.org/10.1007/s40279-013-0135-9

Sutton, K. M., \& Bullock, J. M. (2013). Anterior cruciate ligament rupture: differences between males and females. Journal of the American Academy of Orthopaedic Surgeons, 21(1), 41-50.

https://doi.org/10.5435/JAAOS-21-01-41

Telianidis, S.; Perraton, L.; Clark, R. A.; Pua y.-H.; Fortin, K., \& Bryant, A. L. (2014). Diminished sub-maximal quadriceps force control in anterior cruciate ligament reconstructed patients is related to quadriceps and hamstring muscle dyskinesia. Journal of Electromyography and Kinesiology, 24(4), 513-519.

https://doi.org/10.1016/j.jelekin.2014.04.014

Thomas, A. C.; Villwock, M.; Wojtys, E. M., \& Palmieri-Smith, R. M. (2013). Lower extremity muscle strength after anterior cruciate ligament injury and reconstruction. Journal of athletic training, 48(5), 610-620.

https://doi.org/10.4085/1062-6050-48.3.23

Waldén, M.; Hägglund, M.; Werner, J.; Ekstrand, J. (2011).The epidemiology of anterior cruciate ligament injury in football (soccer): a review of the literature from a genderrelated perspective. Knee surgery, sports traumatology, arthroscopy, 19(1), 3-10. https://doi.org/10.1007/s00167-010-1172-7

Wesley, C. A.; Aronson, P. A., \& Docherty, C. L. (2015). Lower Extremity Landing Biomechanics in Both Sexes After a Functional Exercise Protocol. Journal of Athletic Training, 50(9), 914-920.

https://doi.org/10.4085/1062-6050-50.8.03 
Romero-Moraleda, B.; Cuéllar, A.; González, J.; Bastida, N.; Echarri, E.; Gallardo, J., y Paredes, V. (2017). Revisión de los factores de riesgo y los programas de prevención de la lesión del ligamento cruzado anterior en fútbol femenino: propuesta de prevención. RICYDE. Revista internacional de ciencias del deporte, 48(13), $117-138$. https://doi.org/10.5232/ricyde2017.04803

Wojtys, E. M.; Huston, L. J.; Lindenfeld, T. N.; Hewett, T. E., \& Greenfield, M. L. V. (1998). Association between the menstrual cycle and anterior cruciate ligament injuries in female athletes. The American journal of sports medicine, 26(5), 614-619.

Woo, S. L.-Y.; Abramowitch, S. D.; Kilger, R., \& Liang, R. (2006). Biomechanics of knee ligaments: injury, healing, and repair. Journal of biomechanics, 39(1), 1-20. https://doi.org/10.1016/j.jbiomech.2004.10.025

Yanguas Leyes J.; Til Pérez L., y Cortés de Olano C. (2011). Lesión del ligamento cruzado anterior en fútbol femenino. Estudio epidemiológico de tres temporadas. Apunts Medicina de I" Esport, 46(171), 137-43.

https://doi.org/10.1016/j.apunts.2011.02.006

Zebis, M. K.; Andersen, L. L.; Brandt, M.; Myklebust, G.; Bencke, J.; Lauridsen, H. B., \& Aagaard, P. (2015). Effects of evidence-based prevention training on neuromuscular and biomechanical risk factors for $A C L$ injury in adolescent female athletes: a randomised controlled trial. British Journal of Sports Medicine, (January 2016) 